\title{
Lung Registration with Improved Fissure Alignment by Integration of Pulmonary Lobe Segmentation
}

\author{
Alexander Schmidt-Richberg, Jan Ehrhardt, René Werner, and Heinz Handels \\ Institute of Medical Informatics, University of Lübeck, Lübeck, Germany \\ schmidt-richberg@imi.uni-luebeck.de
}

\begin{abstract}
Accurate registration of human lungs in CT images is required for many applications in pulmonary image analysis and used for example for atlas generation. While various registration approaches have been developed in the past, the correct alignment of the interlobular fissures is still challenging for many reasons, especially for inter-patient registration. Fissures are depicted with very low contrast and their proximity in the image shows little detail due to the lack of vessels. Moreover, iterative registration algorithms usually require the objects to be overlapping in both images to find the right transformation, which is often not the case for fissures.

In this work, a novel approach is presented for integrated lobe segmentation and intensity-based registration aiming for a better alignment of the interlobular fissures. To this end, level sets with a shape-based fissure attraction term are used to formulate a new condition in the registration framework. The method is tested for pairwise registration of lung CT scans of nine different subjects and the results show a significantly improved matching of the pulmonary lobes after registration.
\end{abstract}

\section{Introduction}

Establishing correspondences between two thoracic CT images by intensitybased non-linear registration is a frequent task in various clinical applications. It is required for example for estimating the pulmonary lung motion of patients using $4 \mathrm{D}$ image data or to align two scans of one patient in follow-up studies. Moreover, atlas generation techniques are generally based on registration algorithms to match scans of different patients.

Various approaches for lung registration have been proposed in the past. To compare their performance, extensive evaluation studies have recently been conducted 122 . While they demonstrate a generally high accuracy of the different algorithms, results also indicate that the interlobular lung fissures are often not sufficiently aligned. Murphy et al. state in their study [2] that while "most of the algorithms performed extremely well in terms of both singularities and lung boundary alignment, [...] differences are much more apparent in the fissure alignment category." This has mainly two reasons: First, the fissures are often depicted with very low contrast - especially in low-dose CT images - and 
intensity-based registration is therefore difficult. There are also few vessels in the proximity of fissures, which leads to homogeneous greyvalues in this region. Secondly, to steer iterative algorithms in the right direction, structures usually have to be overlapping. While multi-scale approaches and regularization schemes implicitly address these problems, small structures such as the fissures are not visible on coarse scales and no forces are explicitly generated that align disjunct structures as caused by large displacements or anatomical dissimilarities. This is especially severe for inter-patient registration.

Several recent publications propose combining image registration and a segmentation of the object-of-interest to improve registration in critical regions [34]. However, these methods use solely intensity-based segmentation methods that are not applicable for lobe segmentation. A registration of distance maps generated from lung boundaries and fissure segmentations was therefore proposed in [5], but image intensities are not considered.

In this work, we present an approach for including explicit fissure alignment in intensity-based image registration algorithms. Assuming a known lobe segmentation of one image (e.g. an atlas), automatic segmentation of the second image and registration are incorporated in a joint approach. Their mutual dependency is used to combine intensity- as well as shape information to guide the registration. An integrated variational formulation of the problem is presented. The approach is preliminarily evaluated for pairwise inter-patient registration using nine thoracic CT images.

\section{Methods}

We proceed by briefly introducing an intensity-based non-linear registration scheme (Section 2.1). An approach for automatic lobe segmentation based on multi-object level sets was proposed in [6] and is summarized in Section 2.2. In Section 2.3. these methods are then integrated in a joint framework.

\subsection{Variational Lung Registration}

Given two images $I^{R}$ and $I^{T}$ (called reference and template image) with $I: \Omega \mapsto$ $\mathbb{R}$ and the image domain $\Omega \subset \mathbb{R}^{3}$, the registration problem can be formulated as finding a plausible transformation $\varphi: \Omega \mapsto \Omega$ that transforms the template image to match the reference image by minimizing the energy functional

$$
\mathcal{J}^{\operatorname{Reg}}[\varphi]:=\mathcal{D}\left[I^{R}, I^{T} ; \varphi\right]+\alpha_{1} \mathcal{S}[\varphi] .
$$

Here, $\mathcal{D}$ is a distance measure quantifying the (dis-)similarity between reference and transformed target image. The plausibility of the transformation is controlled by the regularizer $\mathcal{S}$, which smoothes the field and thereby avoids discontinuities like gaps or folding, and $\alpha_{1}$ weights the amount of smoothing.

With regard to the Euler-Lagrange equation of the functional, the transformation $\varphi$ can be optimized using a gradient descent according to

$$
\frac{\partial \varphi}{\partial t}=\boldsymbol{f}(\boldsymbol{u})+\alpha_{1} \mathcal{A} \boldsymbol{u}
$$


where $\boldsymbol{u}(\boldsymbol{x}):=\varphi(\boldsymbol{x})-\boldsymbol{x}$ denotes the displacement field of the transformation, $\mathcal{A}$ is a linear operator related to the regularizer and $\boldsymbol{f}$ a force term derived from the distance measure. Without loss of generality, diffusion regularization and normalized SSD-forces (NSSD) are used in this work [7].

\subsection{Lobe Segmentation with Multi-object Level Sets}

In level set segmentation, the boundary of an object $\Sigma \subset \Omega$ in the image is represented implicitly as the zero-level curve of a level set function $\phi: \Omega \mapsto \mathbb{R}$. Here, $\phi$ is defined as the distance function to the boundary with $\phi(\boldsymbol{x})<0$, if $\boldsymbol{x} \in \Sigma$ and $\phi(\boldsymbol{x})>0$, if $\boldsymbol{x} \in \Omega \backslash \Sigma$. The optimal level set is determined by minimizing the energy functional

$$
\mathcal{J}^{S e g}[\phi]:=\mathcal{E}[I ; \phi]+\alpha_{2} \mathcal{I}[\phi] .
$$

The internal energy $\mathcal{I}$ is defined as in [6] and enforces a smooth surface. The region-based external energy

$$
\mathcal{E}[I ; \phi]:=-\int_{\Omega}(1-H(\phi(\boldsymbol{x}))) \log p_{\text {in }}(I(\boldsymbol{x}))+H(\phi(\boldsymbol{x})) \log p_{\text {out }}(I(\boldsymbol{x})) d \boldsymbol{x}
$$

integrates a-priori knowledge about intensity distributions $p_{\text {in }}$ inside and $p_{\text {out }}$ outside the lungs, respectively, and draws the segmentation to the lung boundaries. The Heaviside function $H$ is used to distinguish inside from outside.

For the minimization with respect to the level set function, the Euler-Lagrange equation is derived and a gradient descent is performed according to

$$
\frac{\partial \phi}{\partial t}=-\delta(\phi)\left(\log \frac{p_{\text {in }}}{p_{\text {out }}}-\alpha_{2} \nabla \frac{\nabla \phi}{\|\nabla \phi\|}\right) .
$$

To simultaneously segment the pulmonary lobes, the level set framework is extended following Brox et al. [8] by employing $N$ functions $\phi_{i}, i=0, \ldots, N-1$, each representing one object $\Sigma_{i}:=\left\{\boldsymbol{x}: \phi_{i}(\boldsymbol{x})<0\right\}$. Front propagation is then performed according to

$$
\frac{\partial \phi_{i}}{\partial t}=-\delta(\phi)\left(e_{i}-\max _{H\left(\phi_{j}\right)<0, j \neq i}\left(e_{j}, e_{i}-1\right)\right) \text { with } e_{k}:=\log p_{k}-\frac{\alpha_{2}}{2} \nabla \frac{\nabla \phi_{k}}{\left\|\nabla \phi_{k}\right\|} .
$$

Here, $e_{i}$ serves as a (mostly outwards-directed) force that affects the function $\phi_{i}$. The final update value is then determined by a competition of this force and the maximal force of all adhering level set functions $\phi_{j}$. For lobe segmentation, we have $N=6$ objects (background and five lobes) and set $p_{0}:=p_{\text {out }}$ and $p_{i}:=p_{\text {in }}$ for $i=1, \ldots, 5$.

Using the model described above, only smoothing is performed between two lobes because $p_{i}$ equals $p_{j}$ in these cases. Therefore, we follow [6] and define an additional term that draws the contour to the fissures. With this purpose, the interlobular fissures are segmented employing the supervised enhancement filter 


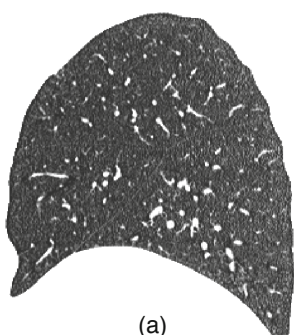

(a)

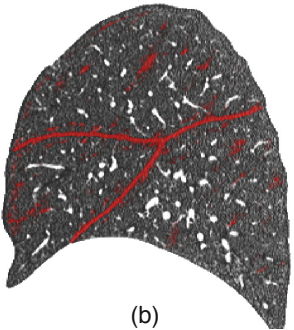

(b)

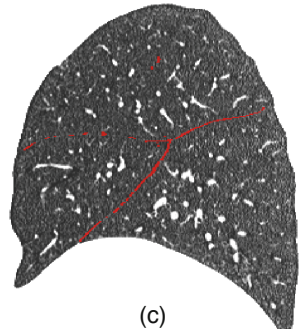

(c)

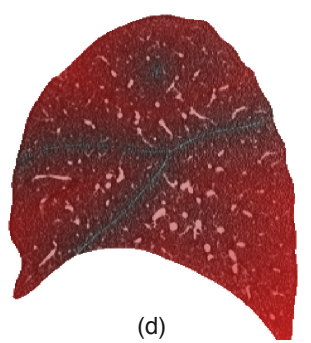

(d)

Fig. 1. Computation of the cost image for lobe segmentation: (a) the CT image $I$; (b) the fissure segmentation as computed by the supervised enhancement filter; (c) the skeleton of the post-processed fissure segmentation (dilated by one voxel to enhance visibility); (d) the cost image $C$, which is incorporated in the force term

proposed by van Rikxoort et al. [9, which uses intensity and shape information to train a kNN classification for the detection of fissure voxels. To reduce false positives, parameters are chosen aiming at a high specificity at the expense of sensitivity. A cost image $C: \Omega \mapsto \mathbb{R}$ is computed as distance transformation to the skeleton $K$ of the fissure segmentation (see Figure 11). With this, the force term in (4) can be reformulated to incorporate a fissure-attraction force by

$$
e_{k}:=\log p_{k}-\frac{\alpha_{2}}{2} \nabla \frac{\nabla \phi_{k}}{\left\|\nabla \phi_{k}\right\|}-\frac{\beta}{2} \nabla \phi_{k} \cdot \nabla C .
$$

\subsection{Integrated Lobe Segmentation and Registration}

Registration and lobe segmentation are combined following the principle idea of 4 and extending it by multi-object lobe segmentation. In the procedure, segmentations $\phi_{i}^{T}$ of the lobes in the template image are assumed to be known. The approach aims at simultaneously finding a transformation $\varphi$ and a segmentation $\phi_{i}^{R}$ of the reference image under the additional condition that the transformed template segmentation resembles the segmentation of the reference image.

The integrated model is defined by the joint energy functional

$$
\mathcal{J}^{\text {Joint }}\left[\varphi, \phi^{R}\right]:=\mathcal{J}^{R e g}[\varphi]+\gamma \mathcal{J}^{S e g}\left[\phi^{R}\right]+\gamma_{1} \mathcal{P}\left[\phi^{T} ; \varphi, \phi^{R}\right],
$$

where the segmentation- and registration-related terms are defined as before and $\gamma$ and $\gamma_{1}$ weight the terms against each other. The shape prior term

$$
\mathcal{P}\left[\phi^{T} ; \varphi, \phi^{R}\right]:=\frac{1}{2} \int_{\Omega} \sum_{i=0}^{N-1} \delta\left(\phi_{i}^{R}\right)\left(\phi_{i}^{T} \circ \varphi-\phi_{i}^{R}\right)^{2} d \boldsymbol{x}
$$

links segmentation and registration by penalizing large distances between the zero level set of $\phi^{R}$ and the transformed template segmentation $\phi_{i}^{T} \circ \varphi$. It is formulated exploiting the fact that the value of a level set function is defined to be the distance to the closest boundary. 

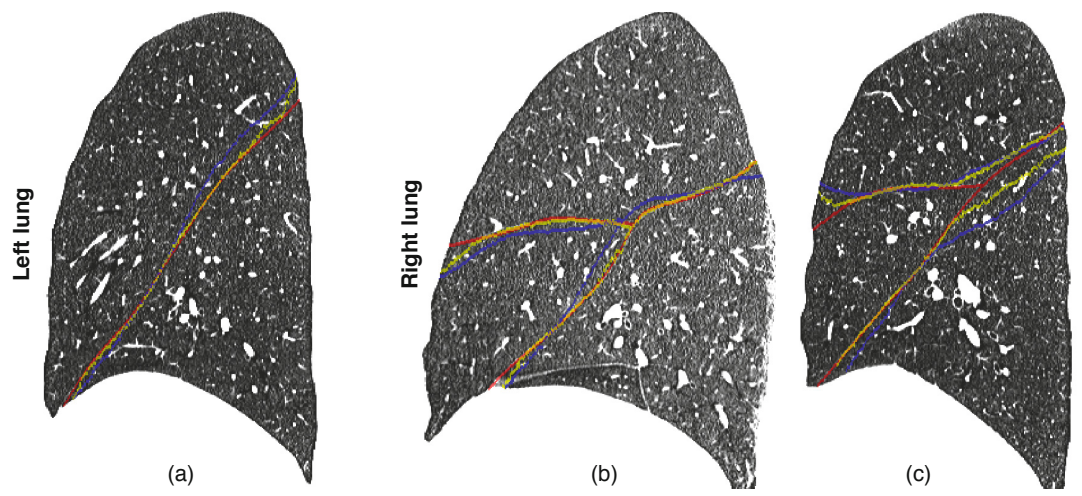

Fig. 2. Reference images with lobe boundaries as color overlay. Red: manual reference segmentation; Blue: template segmentation after registration with $\mathcal{J}^{\text {Reg }}$; Yellow: template segmentation after registration with $\mathcal{J}^{\text {Joint }}$. In the left lung (a), alignment is improved for almost all patients. For the right lung, results depend on the initialization: While fissures are well-aligned in (b), alignment fails with both algorithms in (c).

The joint energy term is alternately minimized in direction of the transformation and of the reference segmentation. Including the shape prior term, the gradient descent equation (2) for the registration part reads

$$
\frac{\partial \varphi}{\partial t}=\boldsymbol{f}(\boldsymbol{u})+\alpha_{1} \mathcal{A} \boldsymbol{u}+\gamma_{1} \sum_{i=0}^{N-1} \delta\left(\phi_{i}^{R}\right)\left(\phi_{i}^{T} \circ \varphi-\phi_{i}^{R}\right) \nabla\left(\phi_{i}^{T} \circ \varphi\right)
$$

where the last term corresponds to the Euler-Lagrange equation of $\mathcal{P}$. It acts as an additional force that affects $\varphi$ to transform the template segmentation in the direction of the reference segmentation. The segmentation is updated according to (4) but using the force

$$
e_{k}:=\log p_{k}-\frac{\alpha_{2}}{2} \nabla \frac{\nabla \phi_{k}^{R}}{\left\|\nabla \phi_{k}^{R}\right\|}-\frac{\beta}{2} \nabla \phi_{k}^{R} \cdot \nabla C+\frac{\gamma_{2}}{2}\left(\phi_{k}^{T} \circ \varphi-\phi_{k}^{R}\right)
$$

with $\gamma_{2}:=\gamma_{1} / \gamma$ for force computation. Here, the additional term prevents the segmentation from diverging too far from the transformed template segmentation and thus avoids leakage into neighboring structures.

\section{Results}

The approach is evaluated for inter-patient registration on a set of nine thoracic normal dose CT images of different subjects $(120 \mathrm{kVp}, 450-750 \mathrm{mAs}, 0.79 \times 0.79 \times$ $0.7 \mathrm{~mm}$ spacing, cropped to lung region). For each image, lobe segmentations $S$ where generated for evaluation using a spline-based interpolation of manually defined fissure points and a subsequent manual correction. To avoid a bias due 
Table 1. Lobe and fissure alignment after registration with common intensity-based registration $\mathcal{J}^{R e g}$ and the proposed registration with fissure alignment $\mathcal{J}^{\text {Joint }}$, averaged over the results of all 72 image pairs. The mean fissure distance is given in millimeter.

\begin{tabular}{|c|c|c|c|c|c|c|c|}
\hline Lobe & $\begin{array}{l}\text { Dice } \\
\mathcal{J}^{\text {Reg }}\end{array}$ & $\begin{array}{l}\text { coeff. } \\
\mathcal{J}^{\text {Joint }}\end{array}$ & Fissure & $\begin{array}{r}\text { Metr } \\
\mathcal{J}^{\text {Reg }}\end{array}$ & $\begin{array}{l}\text { Fiss } \\
\mathcal{J}^{\text {Joint }}\end{array}$ & $\begin{array}{l}\text { Mea } \\
\mathcal{J}^{\text {Reg }}\end{array}$ & $\begin{array}{l}\text { dist. } \\
\mathcal{J}^{\text {Joint }}\end{array}$ \\
\hline \multirow{3}{*}{$\begin{array}{l}\text { left superior } \\
\text { left inferior } \\
\text { right superior } \\
\text { right middle } \\
\text { right inferior }\end{array}$} & $\begin{array}{l}0.92 \\
0.93\end{array}$ & $\begin{array}{l}0.95 \\
0.95\end{array}$ & left & 0.18 & 0.52 & 9.79 & 4.74 \\
\hline & 0.88 & 0.90 & \multirow{2}{*}{$\begin{array}{l}\text { right oblique } \\
\text { right horizontal }\end{array}$} & 0.16 & 0.44 & 11.34 & 6.10 \\
\hline & $\begin{array}{l}0.69 \\
0.91\end{array}$ & $\begin{array}{l}0.73 \\
0.94\end{array}$ & & 0.15 & 0.24 & 14.72 & 12.70 \\
\hline
\end{tabular}

to the choice of the reference image, a pairwise validation is employed, that means each image is registered with the remaining eight images leading to 72 registrations. Parameters of the segmentation and registration components were chosen as specified in 67. The values for the additional parameters $\gamma_{1}$ and $\gamma_{2}$ were optimized empirically. Since the segmentation converges faster than the registration, five registration iterations are performed for each segmentation iteration (this parameter can be seen as related to $\gamma$ in (6) ). In total, computation time lies between 6 min and $14 \mathrm{~min}$, which means a prolongation of approximately $48 \%$ in comparison to the standard registration.

For a quantitative evaluation, the Dice coefficient of the manual reference segmentation $S^{R}$ and transformed template segmentation $S^{T} \circ \varphi$ were calculated after registration with the common approach using $\mathcal{J}^{R e g}$ and the presented registration with integrated lobe segmentation $\mathcal{J}^{\text {Joint }}$ (see Table 1). Since the Dice coefficient may be difficult to interpret due to the varying size of the individual lobes, two additional metrics that quantify fissure alignment are considered: on the one hand, the mean distance of fissure voxels in $S^{T} \circ \varphi$ to fissure voxels $S^{R}$; on the other hand, a metric inspired by Murphy et al. [2] that estimates the proportion of the lobe boundaries in the reference image that lie in the proximity of the lobes in the transformed template image. For this, let $B\left(S^{T} \circ \varphi\right)$ be the set of voxels in the transformed template segmentation $S^{T}$ that lie at the boundary between two lobes. To incorporate some tolerance, this region is expanded by \pm 3 voxels in $z$-direction and denoted by $B^{+}$. The proportion of correctly segmented boundary voxel $X$ can then be quantified by

$$
\operatorname{Fiss}\left(S^{T} \circ \varphi, S^{R}\right):=\frac{\left|\left\{X: X \in B^{+}\left(S^{T} \circ \varphi\right) \wedge X \in B\left(S^{R}\right)\right\}\right|}{\left|\left\{X: X \in B\left(S^{R}\right)\right\}\right|} .
$$

The results confirm a significantly better alignment of the lung fissures using the proposed registration approach (paired $t$-test, $p \leq 0.05$ for all metrics and subjects serving as reference image). Averaged over all registrations, $40 \%$ of the fissures are well aligned using the joint approach, compared to only $16 \%$ with the standard method. Results are better for the left lung (52\%) than for the fissures in the right lung ( $44 \%$ and $24 \%$ for horizontal and oblique fissures). The same observations hold for the mean fissure distance. 


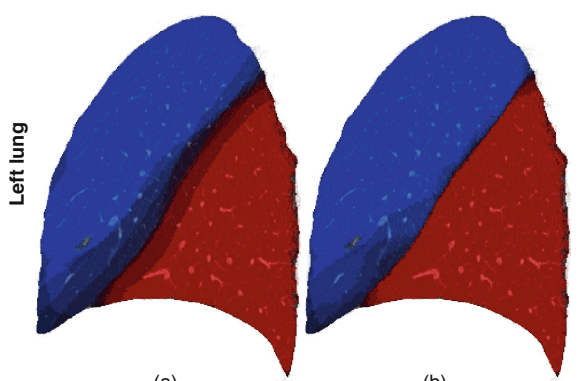

(a)
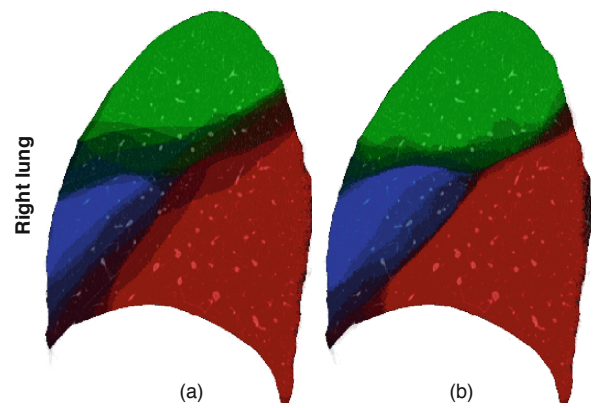

Fig. 3. Transformed lobe segmentations of eight subjects dealing as atlas after registration with the remaining subject. The joint approach (b) produces a sharper and therefore more consistent atlas segmentation than the standard registration (a).

The results are visualized in Figure2 A much better alignment of the fissures is observed in regions where fissures were segmented successfully. Furthermore, in a proof-of-concept demonstration the application to atlas generation is explored and illustrated in Figure 3. Here, lobe segmentations of eight subjects were transformed to the remaining subjects and summed up. The joint registration approach provides a much sharper segmentation of the reference image, which indicates a superior matching of the lobes.

\section{Discussion}

The results show that integrating lobe information in intensity-based registration can improve fissure alignment considerably. Employing level set methods for this entails several advantages over - for example - directly considering the cost image $C$. First, forces are only generated along the zero level set, that means only at the fissures and not at places wrongly classified as fissures due to noise. Gaps in the fissure segmentations are not exceedingly critical since they are automatically bridged by the level set framework. If no fissure information is present in an image region, no forces (beside smoothing) are generated that move $\phi^{R}$ away from $\phi^{T}$ and thus only the standard registration is applied in this area. This effect could be enhanced by truncating $C$ to restrain the influence of the fissure alignment to a smaller range, for example if segmentation is suffering from bad image quality.

Problems arise in particular in the right lung between horizontal and oblique fissures because the level set can be attracted by the wrong fissure if the initialization is bad. Moreover, the fissure enhancement filter performs worse in this region. A more precise initialization - for example using an anatomical atlas [6] - could improve the results in this area.

Another interesting application of the approach would be the estimation of breathing motion in $4 \mathrm{D}$ image data. While the displacement is smaller in this case, fissure segmentation is very difficult in $4 \mathrm{D}$ images due to the often lower 
radiation dose per image as well as motion and reconstruction artifacts. These aspects will be the focus of future investigations.

\section{Conclusion}

In this work, an approach is presented to include pulmonary lobe segmentation in intensity-based registration with the aim of improving the alignment of the interlobular fissures. Segmentation is done using multi-object level sets with an additional shape-based fissure attraction term. This information is used in a new condition in the registration framework to match the lobes to each other.

The approach was tested for inter-patient registration on nine thoracic CT scans. A considerably better alignment of the fissures was observed but the method depends on a reasonably good fissure segmentation. Moreover, huge anatomical differences between the patients imply a bad initialization of the segmentation, which may result in a misalignment of the fissures. This is especially critical for the right lung, where horizontal and oblique fissures are close to each other.

Acknowledgements. This work is supported by the German Research Foundation DFG (EH 224/3-1).

\section{References}

1. Brock, K.K.: Deformable Registration Accuracy Consortium: Results of a multiinstitution deformable registration accuracy study (MIDRAS). Int. J. Radiat. Oncol. Biol. Phys. 76(2), 583-596 (2010)

2. Murphy, K., van Ginneken, B., Reinhardt, J.M., Kabus, S., Ding, K., et al.: Evaluation of Registration Methods on Thoracic CT: The EMPIRE10 Challenge. IEEE Trans. Med. Imag. 30(11), 1901-1920 (2011)

3. Yezzi, A., Zöllei, L., Kapur, T.: A variational framework for integrating segmentation and registration through active contours. Med. Image Anal. 7(2), 171-185 (2003)

4. Schmidt-Richberg, A., Handels, H., Ehrhardt, J.: Integrated segmentation and nonlinear registration for organ segmentation and motion field estimation in 4D CT data. Methods Inf. Med. 48(4), 344-349 (2009)

5. van Rikxoort, E.M., Prokop, M., de Hoop, B.J., Viergever, M.A., Pluim, J.P.W., van Ginneken, B.: Automatic Segmentation of Pulmonary Lobes Robust against Incomplete Fissures. IEEE Trans. Med. Imag. 29(6), 1286-1296 (2010)

6. Schmidt-Richberg, A., Ehrhardt, J., Wilms, M., Werner, R., Handels, H.: Pulmonary Lobe Segmentation with Level Sets. In: Haynor, D.R., Ourselin, S. (eds.) Proc. SPIE, p. $83142 \mathrm{~V}(2012)$

7. Schmidt-Richberg, A., Ehrhardt, J., Werner, R., Handels, H.: Diffeomorphic Diffusion Registration of Lung CT Images. In: van Ginneken, B., Murphy, K., Heimann, T., Pekar, V., Deng, X. (eds.) Medical Image Analysis for the Clinic: A Grand Challenge, MICCAI 2010, pp. 55-62 (2010)

8. Brox, T., Weickert, J.: Level Set Segmentation with Multiple Regions. IEEE Trans. Image Process. 15(10), 3213-3218 (2006)

9. van Rikxoort, E.M., van Ginneken, B., Klik, M., Prokop, M.: Supervised Enhancement Filters: Application to Fissure Detection in Chest CT Scans. IEEE Trans. Med. Imag. 27(1), 1-10 (2008) 